\title{
Intelligent Coordination Distribution of the Whole Supply Chain Based on the Internet of Things
}

\author{
Hongxiu Cui (i) \\ School of Business, Changchun Sci-Tech University, Changchun, Jilin 130600, China \\ Correspondence should be addressed to Hongxiu Cui; 100231@cstu.edu.cn
}

Received 20 January 2021; Revised 23 February 2021; Accepted 8 March 2021; Published 17 March 2021

Academic Editor: Wei Wang

Copyright ( $) 2021$ Hongxiu Cui. This is an open access article distributed under the Creative Commons Attribution License, which permits unrestricted use, distribution, and reproduction in any medium, provided the original work is properly cited.

\begin{abstract}
In this paper, through the intelligent research of the whole process of logistics and distribution with the Internet of Things supply chain, we study how to improve the development of the cold chain, reduce the loss in circulation, improve the social and economic benefits, and carry out intelligent information collection, monitoring, management, and information tracing of the whole cold chain. This paper analyzes and empirically studies the impact of key technologies of the Internet of Things in cold chain coordination from the perspective of building an intelligent cold chain coordination system with the Internet of Things technology. This paper analyzes the current situation of cold chain logistics and the impact that the application of IoT technology will have, explains that IoT technology can improve the intelligence level of the cold chain, and then introduces the application of intelligent cold chain logistics under IoT orientation, combining the process of cold chain logistics with the three-layer architecture of IoT technology. By extracting the key technologies of IoT perception layer, network layer, and intelligence layer, the intelligent cold chain coordination system based on IoT technology is constructed, and then, the correctness of the system is verified, to have some reference and evaluation for the cold chain construction. The system was then verified to have some reference and guidance significance for the construction and evaluation of the cold chain. The results of this paper are more accurate and more efficient.
\end{abstract}

\section{Introduction}

The combination of artificial intelligence, big data technology, Internet of Things technology, "Internet +" technology and coordination technology has enabled modern coordination technology to develop in the direction of intelligent development and promoted the update of intelligent coordination technology [1]. The intelligent coordination system based on information technology coordinates the perception of the system in warehouses, distribution and information management through timely analysis and processing of organizational information. Smart organization is concerned with uniting the Internet of Things, WSN, and today's Internet to form a controlled, intelligent, and scientific coordination through real-time, effective, and scientific management, deepening resource utilization and productivity and providing a more socially valuable coordination effect [2]. Logistics technology involves various fields and covers logistics processes such as management, inventory, and distribution; on the other hand, the vigorous development of Internet of Things technology and the coordinated management of intelligent complete systems have produced a good soil for the traditional logistics technology to grow [3]. Today, where people's basic living needs have been met, people are focusing more on improving the quality of life [4]. Consumers and retailers, in general, are paying increased attention to issues such as safety and freshness of food; as an important part of the food, its safety and contamination issues need to receive more attention [5]. From the release of a series of documents, the state is highly concerned about the safety issues of the people and the further implementation of the national safety and security strategy [6]. The government will be committed to solving the problems brought to people's lives because of safety issues, and reduce the economic losses caused by it [7]. With the growing demand for freshness, the traditional means of coordination and transportation are far from being able to meet people's needs, and in this context, the market 
demand for cold chain coordination is increasing [8]. As the traditional coordination means cannot guarantee the quality in the process of transportation, it is easy to cause a lot of loss, which leads to the rise of price and increase the cost of transportation [9].

\section{Related Works}

Mahmud et al. studied the design of smart logistics system in the environment of e-commerce and found that a good smart logistics system can shorten the time cycle of the supply chain and also save a lot of costs, and also can use accurate and dynamic management methods to accelerate the rapid development of e-commerce [10]. Pagan et al. pointed out that RFID's Internet of Things technology can be used for the auxiliary management of decision support in the pan-study intelligent logistics system, mainly including regional logistics index prediction, multimodal transport decision support and geographic information system decision-making. They designed a multimodal transport decision support model and analyzed the process and functions of GIS decision-making [11]. Opiyo used a comprehensive evaluation method to build a comprehensive evaluation system for the wisdom logistics system and proposed a wisdom logistics path optimization model based on a planning algorithm, which optimized the path selection and improved the function of the wisdom logistics system [12]. Uggla et al., for the wisdom cloud coordination system, analyzed the incentive model of information sharing and proposed an incentive allocation mechanism and supervision mechanism [13]. Luo and Wood analyzed the factors affecting customers' use of wisdom logistics platform based on technology acceptance model, identified key factors and influence paths, and proposed suggestions for optimizing wisdom logistics information platform [14]. Firouzi et al. studied the urban underground wisdom logistics system's architecture, network layout, and management mode, and established an intelligent monitoring system and optimized the service efficiency and operation cost through information integration and intelligent decision-making [15]. A service robot was designed for the smart supermarket [16]. The service robot builds a map of the supermarket through positioning and mapping functions to obtain the location of goods, and when it receives a request to grab goods, it immediately performs path planning to optimize the moving distance of the task and can help customers to take goods from the shelves and also stop to answer questions from customers during a patrol [17]. The service robot system enables comfortable and efficient shopping in a smart supermarket.

Based on the basic theories of the Internet of Things and smart coordination, this paper proposes the use of RFID technology to successfully build a smart supermarket. Based on an in-depth analysis of the principles and components of the smart supermarket, the biggest problem in the smart supermarket is the problem of RFID tag collision, and the RFID tag anti-collision algorithm is studied. In logistics enterprises that already have information systems, the logistics distribution centre for a city, including the primary and secondary distribution centres, is not perfect in order management, warehouse management, distribution management, and other logistics aspects of resource integration capabilities, and the transport tracking and route planning and other functions need to be improved. Optimizing the path in the coordination and distribution system is the most important part of coordination and distribution, and optimizing the coordination and distribution path can improve the distribution efficiency. Many research experts have conducted research based on the forbidden search algorithm, simulated annealing algorithm, and genetic algorithm to apply for the optimization of logistics distribution path, but each algorithm has its limitations and needs to be improved and optimized or combined with other algorithms to study the path optimization problem. To analyze and study an improved genetic algorithm applied to the path optimization in the distribution link of a logistics system, firstly, a mathematical model is constructed according to the problem description. Then, the chromosomes with the best fitness are generated in the population from one generation to another through the operations of selection, heredity, and mutation, and each generation is subject to superiority and inferiority until the convergence conditions are satisfied. The algorithm is then calculated and validated by simulation based on the experimental data.

\section{Intelligent Analysis of Supply Chain Coordination Based on the Internet of Things}

3.1. Intelligent Coordination System Design and Key Technologies. Using intelligent technology as a platform, by imitating human thinking, learning, reasoning and other intelligent behaviors, the coordination system can analyze and make decisions on its own to solve certain problems in the coordination system. It is a kind of intelligent coordination [18]. Figure 1 shows the overall structure of the intelligent coordination system. In general, the intelligent logistics system uses bar code, radio frequency identification technology, sensors, and other technologies to identify and collect logistics information, and then stores and performs basic operations through the microprocessor in the embedded system, and then the logistics system platform in the scheduling layer manages various logistics information, including order information, inventory information, and distribution information, and at the same time adds the application of algorithmic decision-making, and then transmits the information through the wireless network. The information is then transmitted to the distribution service personnel through wireless networks and the distribution vehicles are located through GPS technology, thus realizing efficient and optimal management of the logistics system, which not only reduces logistics costs and resource consumption but also reduces carbon emissions, thus making it more friendly to the environment [19].

The express life cycle is generally as follows: the general cargo will first arrive in a city distribution centre, the distribution centre for initial sorting; some cities will be divided into regions, so that the entire distribution centre will be divided only to each district, pulled to each district 


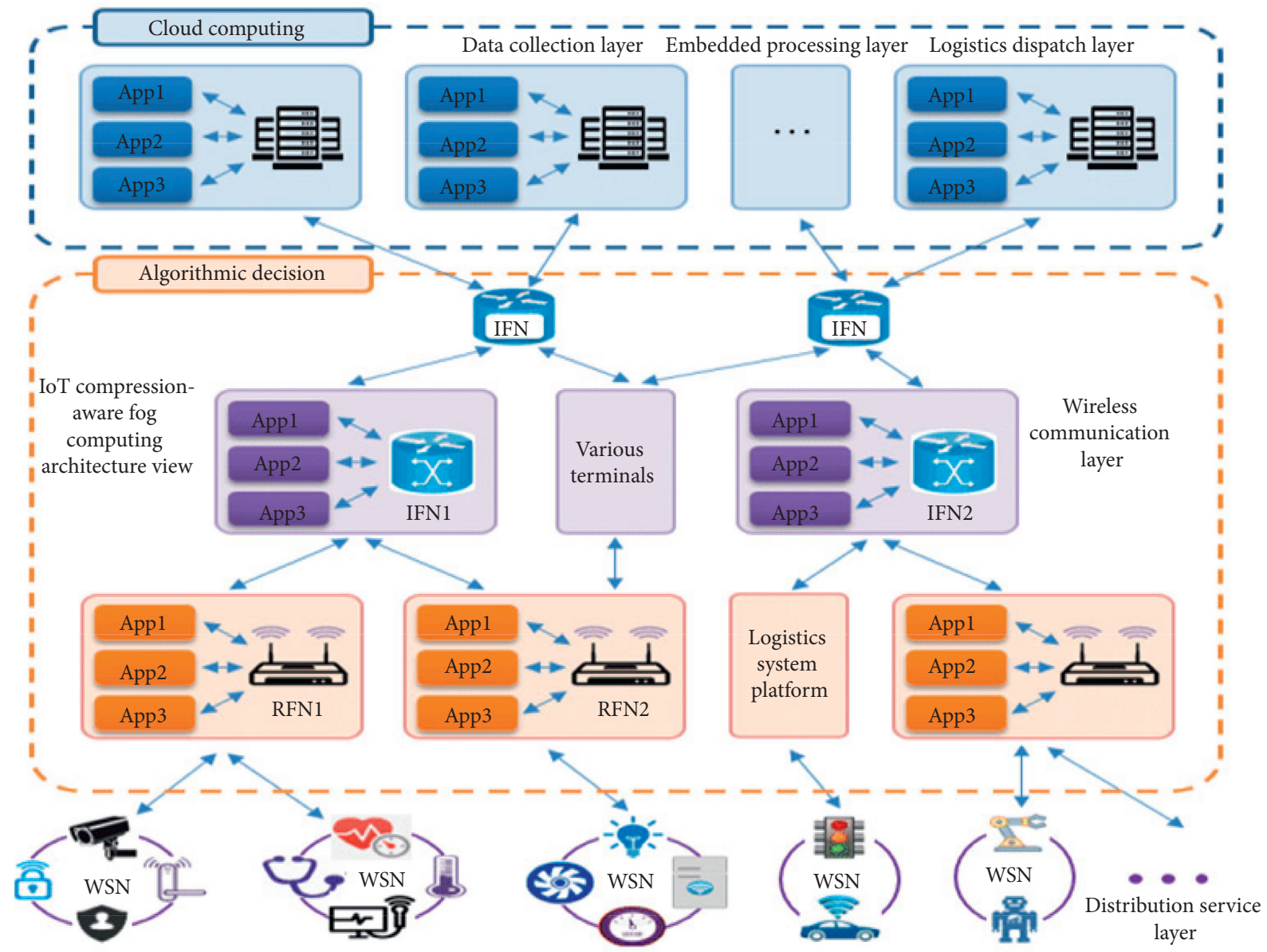

FIGURE 1: The overall structure of the intelligent coordination system.

distribution centre, and then divided into several parts according to the courier's delivery range [20]. The coordination information system of a comprehensive coordination system should integrate the information flow of each different business operation function, including the management aspects of orders, distribution, inventory, and human resources.

With the rapid development of online shopping, the coordination in terms of e-commerce has characteristics, such as the large scale of cargo volume and obvious peaks and valleys, which render the e-commerce coordination distribution different from other distribution centres. E-commerce distribution centres are generally built on a large scale, with many storage sizes, and pay great attention to the improvement of inventory and distribution efficiency, usually setting up a central distribution centre, a regional distribution centre, and a secondary distribution centre in a city.

The professional coordination distribution centre is dominated by third-party coordination enterprises. This kind of distribution centre has two important characteristics-one is to have a relatively high transportation and distribution capacity, and the other is a more suitable geographical location. From these two points, the goods can be delivered to the users promptly. This kind of distribution centre only provides coordination services, mainly inventory management, and transportation and distribution services, and the goods in the distribution centre still belong to the producer or other providers who produced them; therefore, the modernization of this kind of distribution centre tends to be a higher priority [21]. The problem of distribution centre planning is the problem of carrying throughput capacity. In the case of order overflow during the double 11 and other preferential activities, the most central aspect is the reserve of personnel and infrastructure equipment in the distribution centre and the receiving and dispatching outlets. During this period, the distribution centre operates 24 hours without stopping, and how the set can maintain efficient and accurate distribution depends on the reasonable and efficient dispatching of each centre.

One of the primary tasks of distribution centre operations is punctuality, with high time requirements to deliver a specific product to the customer at the time specified by the customer. For the general distribution situation in the city, it is considered that it is affected by the traffic conditions in the city at different times of the day, and these conditions are likely to vary from day to today. Also, another distinctive feature of transport vehicles is that they must spend more time stopping compared to the time they spend traveling. This is because it is not only necessary to load the goods to 
be transported in time before the goods depart, but some unexpected situations may be encountered during transportation, such as traffic jams or road construction. And, when it is delivered to the customer on the vehicle, it must be stopped and unloaded. If the customer has no place to store the goods for the time being, sometimes they will wait for the unloading due to the customer's reasons. In summary, many times during the distribution process of the distribution center, it is impossible to schedule or predict. Sometimes we can only rely on the experience of the distribution center manager to guide the distribution operation activities, as shown in Table 1.

First, the intelligent coordination information management system is researched and designed to have not only the working process and related functions of integrating each link in the coordination distribution process to improve the efficiency of coordination distribution. Then, an improved genetic algorithm is added to the logistics distribution system path optimization to optimize the distribution path and select the optimal distribution route to achieve the goal of shortening the transportation distance and reducing the time and cost spent on logistics at the time of logistics distribution tasks, in addition to the system viewing the detailed status of logistics distribution tasks at any time. So, this coordination system should not only realize the centralized management and sharing of coordination management but also control, analyze, and involve in decision-making.

\subsection{Analysis of Intelligent Coordination Distribution} Algorithms. In recent years, due to the spurt in e-commerce prosperity and high-intensity competition driven by the upgrade of coordination systems, the key problem is that the existing coordination management system software still needs to be improved on the spatial aspects of processing ability, whether it is in the analysis of the geographical location of distribution customers or the selected range of distribution centres, or the route selection in distribution management. Especially in the distribution of goods, how to overcome other uncontrollable factors such as various transportation and weather, and deepen the changes and upgrades of the coordination system are issues of great concern to people [22]. With the situation of the complexity of traffic roads and the diversification of coordination market demand in a twopronged way, a new kind of distribution centre is born following the trend, which is a kind of distribution centre more focused on a certain coordination function. The object of the intelligent coordination system service in this paper is such a distribution centre that focuses on inventory and distribution. The transportation cost of such a distribution centre will occupy a large proportion of the total cost; so, many enterprises with advanced consciousness spare no effort to increase research and investment in distribution management to maintain a certain share in the market. To achieve a truly intensive and intelligent coordination distribution model, it is necessary to realize information sharing and information interaction between supply chain enterprises. The link between enterprises is a project, and all interactively shared information is generated around the project. Therefore, the Internet of Things technology with item status information as the main flow is exactly the key to constructing intelligent coordination distribution covering the entire supply chain. Through the Internet of Things technology, it is possible to achieve seamless integration of information between supply chains, instant communication of status, and instant collaboration of actions, thereby building a unified coordination distribution service information platform, which provides an intelligent distribution service model for the entire supply chain.

This, of course, translates into improvements in specific route selection, so vehicle path planning in distribution is a top priority in coordination systems. Then, the problem of choosing the distribution path so as to reduce the dispatch of vehicles and the allocation of personnel becomes an urgent problem for experts. The content of the path planning problem is mainly to describe a certain number of customers, and the number of commodity needs is also different. The distribution center organizes appropriate travel routes to deliver the goods to the next station to deliver the goods. And according to specific restrictions, such as the minimum mileage required, the minimum cost, and the minimum time, it can meet customer needs and bring huge revenue and value to logistics enterprises.

$$
M=\left[\begin{array}{cccc}
m_{11} & m_{12} & \cdots & m_{1 m} \\
m_{21} & m_{22} & \cdots & m_{2 m} \\
\cdots & \cdots & \cdots & \cdots \\
m_{m 1} & m_{m 2} & \cdots & m_{m m}
\end{array}\right] .
$$

The expert scores the relative importance of the elements on a scale of 1-9 and obtains a judgment matrix between the elements. The meaning of the judgment matrix is the judgment value of a two-by-two comparison of the importance of other related indicators relative to a certain indicator. When the tag enters the effective recognition range of the reader and receives the instruction to read information from the reader, the tag is activated and will randomly select a time slot to send its data information to the reader for data transmission. If more than one tag sends data information to the reader for data transmission in the same time slot, a tag collision will occur, causing any tag information to be unsuccessfully read by the reader. If the tags send data information to the reader at the same time, the collision is complete. If the moment is different, it is a partial collision. The reader sends a feedback command and the colliding tags again transmit data in a randomly selected time slot.

$$
P(n)=\frac{(\lambda t)^{n+1} e^{\lambda t}}{n !}
$$

Assume that a tag needs to occupy a time slot of length T0 to transmit data information, the average value of tags responding to the reader per unit time is $\lambda$, and $G$ denotes the input load. The probability that only one tag responds to the reader within $T$ is 
TABLE 1: Scaling scale.

\begin{tabular}{lcc}
\hline Number & Description & Value \\
\hline 1 & Type, packaging, quality, volume. & 2 \\
2 & Model, load capacity, vehicle volume, transportation distance. & 4 \\
3 & Distribution demand, distribution frequency, distribution point. & 2 \\
4 & Vehicle driving distance, number of vehicles used, delivery time limit. & 4 \\
5 & Meet time window requirements, meet customer distribution needs. & 5 \\
6 & Distribution centre to customer path, customer to customer path. & 7 \\
7 & Gathering goods, completion of vehicle distribution tasks. & 6 \\
\hline
\end{tabular}

$$
P=e^{-2 \pi T^{2}}=e^{-2 G}
$$

Assume that $S$ denotes the algorithm throughput rate, i.e., the number of tags whose messages are successfully read by the reader within a period of $t$. $S$ is

$$
S=G P_{e}=G e^{-2 \pi T^{2}} .
$$

Because each tag to be identified can only uniquely select a time slot in each frame to transmit information to the reader, the probability of collision is reduced. $L$ is the frame length, the number of tags is $d$, and the probability that a tag will randomly select a time slot in a frame length to respond to the reader is

$$
p=\frac{2}{L+d}
$$

According to the principle of the binomial distribution theorem, the probability that $m$ tags are responding to the reader at the same time in each time slot is

$$
\begin{aligned}
P\{X=m\} & =A_{N}^{m}\left(\frac{2}{L+d}\right)^{m}\left(1-\frac{2}{L+d}\right)^{N-m}, \\
S & \left.=\frac{E(m=1)}{L}=\frac{N}{L}\left(1-\frac{2}{L+d}\right)\right)^{N-m}, \\
L & \approx \frac{1-(1 /(L+d+N))}{1+(1 /(L+d+N))} .
\end{aligned}
$$

It can be concluded that the throughput rate $S$ of the algorithm can achieve a maximum value of 0.368 when and only when $N L=0$ and that $S$ is related to the value of the frame length $L$, which varies with $L$. This is shown in Figure 2.

The deterministic tag anticollision algorithm is based on the principle of binary trees. The algorithm divides the collision labels into two subsets of 0 and 1 . If a collision still occurs in a subset, the subset is divided into two subsets of 0 and 1 again, and the cycle continues, and once a collision occurs, it continues to split into two smaller subsets, until a small branch at the bottom is successfully identified, then it returns to another subset of the previous small branch to continue to identify the labels. All these steps are repeated for the entire binary tree until all tags are successfully read. This type of algorithm guarantees that every tag is recognized and is therefore a deterministic tag collision prevention algorithm.

Based on the state-transformed matrix, the Viterbi algorithm based on the dynamic programming principle can

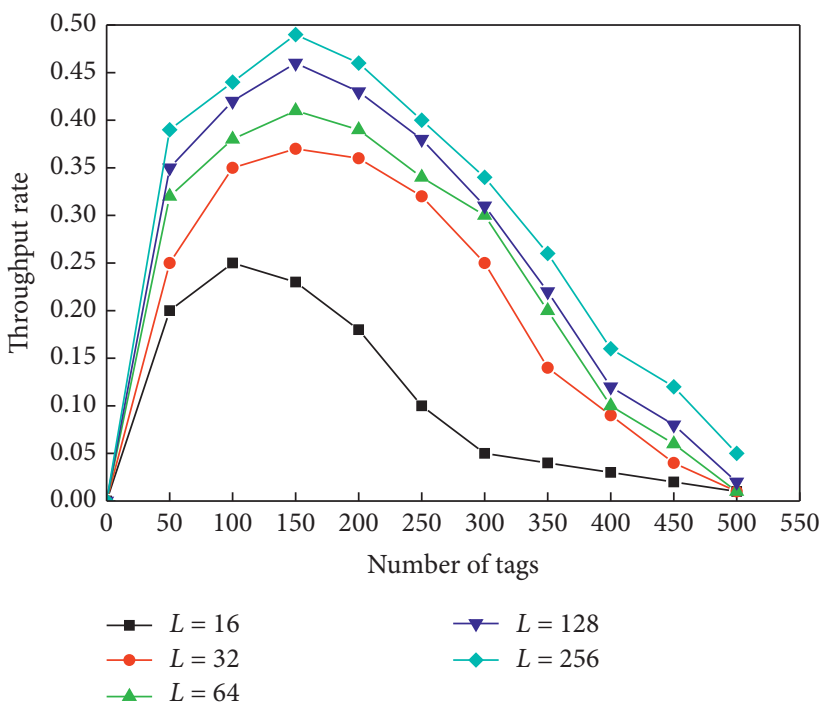

FIgURE 2: Frame length vs. throughput rate.

then be used for candidate path selection [23]. First, assume an HMM model with the following model probability mechanism: assume that the system is in one state in a finite set of states at any discrete time unit. Each state in observation has a fixed probability distribution (eigenvector), which is usually described by a joint Gaussian distribution model, and transitions between states with a fixed probability, the value of which depends on the state the system was in in the previous time unit (Markov chain one-step transfer probability). The functionality of the model depends on the number of states that are abstracted and that are not visible by themselves. Therefore, in the algorithm, the number of states is chosen as a parameter, and each point to be passed in the path is in one of a finite sequence of states, and each point transitions between them with a fixed probability that depends on the state between two adjacent points.

3.3. Experimental Design. For a long time, supply chain managers have suffered from the lack of effective management methods and technical tools to achieve scientific decisions and plan to guide the business to achieve the best operation, such as which production or operation points should be supplied with goods in the warehouse? How much and in what way is the best supply? If new products are produced, how to allocate existing supplier capacity or find new supplier capacity to support the target capacity? How much inventory should be stocked in advance to meet 
seasonal, holiday, and emergency needs? The above questions simply cannot be solved if you try to use manual methods or simple calculations. To make optimal decisions on these issues, it is necessary to have big data and the Internet of Things as the basis to accelerate the flow of information, coordination, and capital in the production process, such as the use of bar codes, radio frequency identification, mobile terminals, and other Internet of Things technology applications to enhance warehouse guidance, operational reminders, and information collection functions to improve warehouse picking, distribution efficiency, and operational quality [24]. Contract documents (arrival order, delivery order, and quality assurance order) use electronic signature and signature technology, based on the information platform, to realize the full electronification of documents and accelerate the flow of contracts and settlements.

The mobile application app is used to improve the convenience of business processing such as material supply plan sharing, arrival and acceptance processing, and performance evaluation, and to enhance the efficiency of employees. Using BI analysis to refine data, we can simulate and optimize the entire supply chain network. The objects of simulation and optimization include projects, routes, processes, products, warehouse nodes, etc., besides the business related to the composition of these elements, which can be either single-objective or multi-objective optimization, to be able to respond quickly to uncertain business and optimize the adjustment strategy. Therefore, emerging information technology can enhance the dynamic configuration of the supply chain; strengthen penalties for supplier quality problem and evaluation management; increase the pursuit of responsibility for equipment quality problems, severely punish suppliers with quality problems, and improve the closed-loop processing mechanism for equipment quality problems; strengthen the penalties for fraudulent qualification performance, substandard quality, serious quality problems, and integrity problems; and apply new technologies to share penalty information, and improve the cost of supplier breach of trust and breach of contract. According to the scope of responsibility management, the professional department will quantitatively evaluate the supplier in terms of manufacturing, on-site installation, operation and maintenance. We make full use of big data technology, establish an evaluation model, and establish a comprehensive evaluation system covering multiple levels, including the supplier's production scale, technical level, quality system, sales service, etc. We apply the evaluation results to the future bidding and procurement process, as shown in Figure 3.

Establish a unified coordination mechanism for material supply, and strengthen the company's key projects, key materials, key equipment material supply process control, and coordination. For problems such as the concentration of supply during the peak period of power grid construction, it is necessary to proactively remind the construction management department to intelligently optimize the project construction timing and delivery cycle to prevent stacking "grabbing". In response to the problem of supplier capacity and transportation tension, it is necessary to strengthen the intelligent prediction of capacity and transportation capacity to ensure orderly staggering; strengthen communication and interaction with major suppliers and carriers, and establish strategic partnerships to ensure sufficient capacity. For major political power protection, people's livelihood projects, and industry expansion and other emergency project needs, ensure the application of procurement and supply "green channel" to meet emergency needs, explore the establishment of an appropriate amount of general supplies' centralized reserve, accelerate the dynamic turnover, and enhance the transfer capacity.

In the specific distribution execution, we manage the distribution plan for the delivered materials and combine big data, mobile applications, and artificial intelligence technology to realize intelligent recommendations of the distribution plan [25]. En-route monitoring equipment realizes en-route monitoring of key materials, early warning of transportation risks and estimation of arrival time to ensure punctual and safe delivery of materials. For example, through the positioning of chips, the transportation monitoring system can accurately grasp the location information of coordination vehicles, plan the route of distribution, and accurately estimate the arrival time of supplies. The full sharing of online and offline data realizes the efficient docking of supply and demand. At the same time, the transportation monitoring system also has an automatic warning function. The system automatically analyzes the transportation status and sends transportation abnormality warning information through data such as the transportation speed, equipment status, and residence time of the materials.

In the distribution and settlement, the contract is the main line. Electronic signature and electronic signature technology are applied to realize the online electronic flow of the whole process of contract signing, contract modification and contract settlement, and realize the timely sharing of contract/change information and intelligent reminders [26]. In terms of waste, it is necessary to break the limitations of materials and asset attributes, establish a cross-regional and cross-professional physical unified deployment platform, establish a cross-regional deployment mechanism for the withdrawal of decommissioned equipment, and rationally allocate available resources in the east, east, and central regions.

The value creation capability of the Intelligent Decision Centre is to be fully utilized to support the company to build a world-class energy Internet enterprise with excellent competitiveness. All-round evaluation of equipment quality, linkage research on grid planning classification, equipment classification, and supplier classification should be carried out to help the company implement accurate investment strategies, and build an intrinsically safe grid. In-depth analysis of the time consumption and cost of each business link is required to carry out quantitative data-driven lean management improvement, to help the company's management transformation, and to aid upgrading [27]. The company's industrial units should be empowered to carry out analysis of manufacturing costs, product quality, market positioning, and other operational activities to help them 


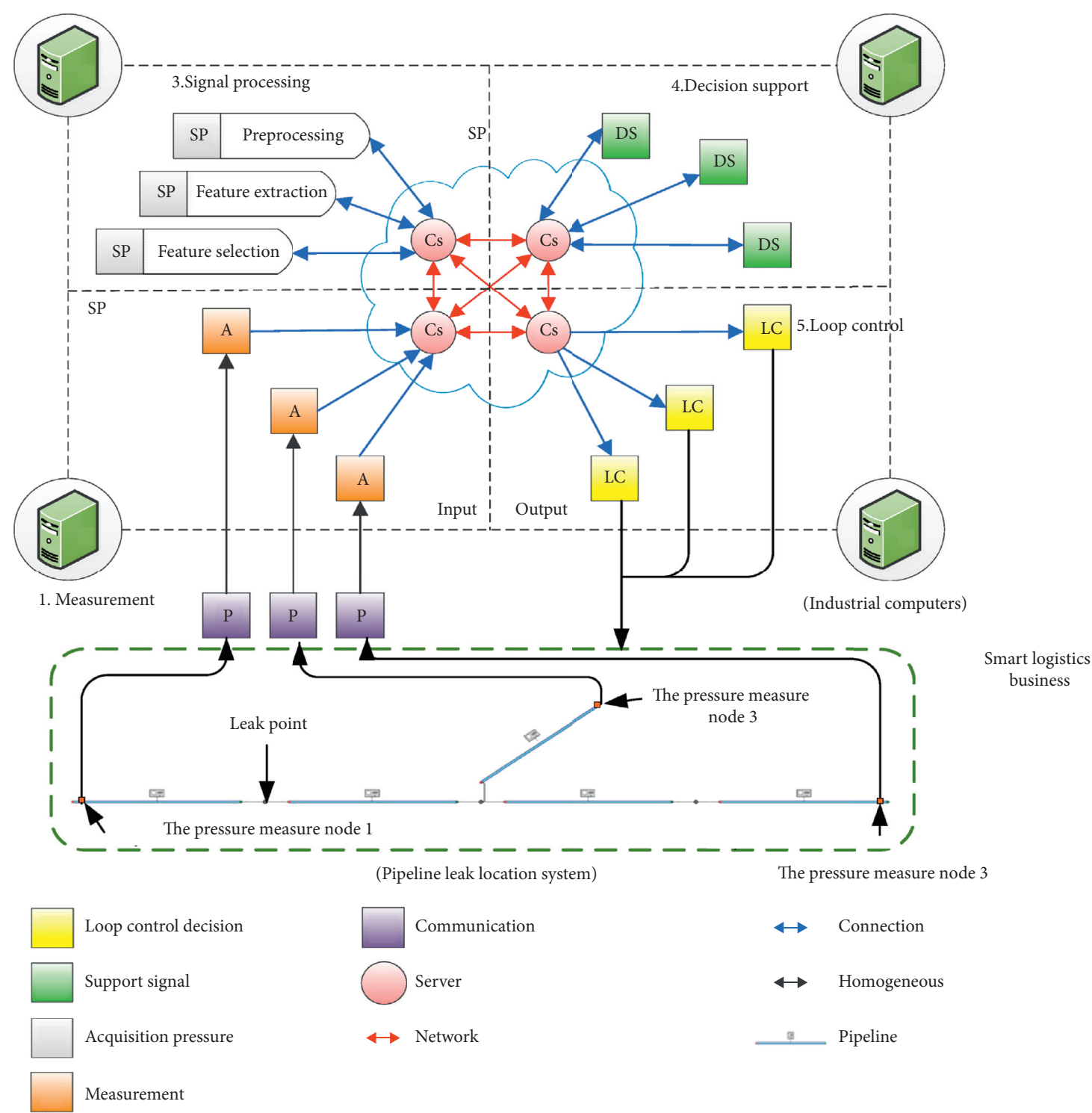

Figure 3: Smart logistics business.

reduce manufacturing costs, improve product quality, and enhance market competitiveness. The company's financial unit should be empowered to carry out analysis of supplier big data, innovate the service mode of combining industry and finance, provide comprehensive and multilevel financial services for suppliers in the energy industry supply chain, help the company expand new profit growth points for market-oriented business, and avoid some suppliers from affecting the supply of materials for power grid construction due to financing difficulties and expensive financing.

\section{Results and Analysis}

4.1. Analysis of Intelligent Distribution Results. Based on the truck number, all the historical routes of the truck are taken out from the software base platform and sorted according to the frequency of transportation in the recent year. For the convenience of elaborating the scheme, it is assumed that the vehicle's routes have not had duplicate origins, that is, the vehicle has transported through four routes in the recent year, called R1, R2, R3, and R4. Taking route R2 as an example, it is assumed that its starting and ending points are $\mathrm{S} 2$ and T2, respectively, and the relationship of the points is shown in Figure 4.

Next, a circle of radius RS is made with the starting point $\mathrm{S} 2$ as the centre. The grid points inside this circle are GP2, GP6, and GP7, so the route R2 will radiate as the route matching fraction from GP2, GP6, and GP7 to the end of the route $\mathrm{T} 2$, and the fraction decays with the distance from the grid point to the point S2. The radiation process is shown in Figure 4. According to the rule, three starting grid points GP2, GP6, GP7 are taken out, and since the endpoint T2 is closest to grid point GP16, GP16 is taken out as the end grid point.

When a truck delivers goods from point A to point B, there is a high probability that it will take another order 


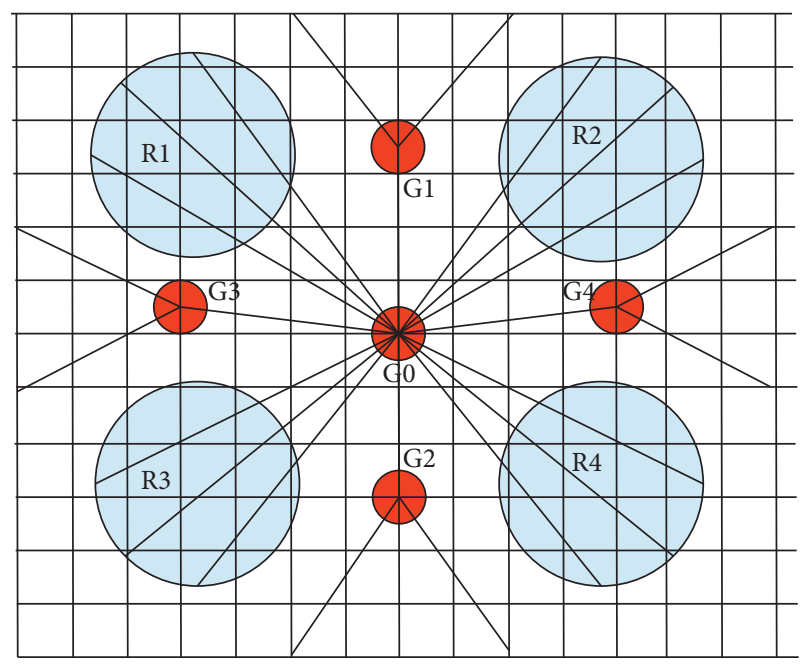

FIGURE 4: Route radiation process.

from point $B$ to point $A$. This order is called a return order. In the process of dispatching the waybill and the corresponding return order, it can be divided into the waiting stage, dispatching to the source stage, starting stage, delivery stage, return order starting stage, and return order delivery stage. When the truck is in the waiting stage, to save costs, the truck will not start, and the driver will find the waybill that can be dispatched to various freight platforms and related communication groups. Most drivers tend to complete the waybill and backorder transactions at this stage in order to avoid the return journey caused by the lack of stock due to no empty goods, so this time is the best time to recommend the waybill and return. In order to facilitate the transaction, the platform data view, the trajectory of the truck and the stopping time can be used to determine whether the truck is in this stage, as shown in Figure 5.

As you can see in Figure 5, the truck data synchronization task runs once the program starts. The route synchronization task runs with the rule that a new task starts immediately after the previous one ends, so there is a situation where all the tasks are connected. The frequency of the waybill data synchronization task runs differently before and after 6 p.m., from once every 60 seconds to once every 600 seconds.

The results of the questionnaire derived from the expert method are entered through Pairwise Comparisons in the Assess/Compare module of the software, as shown in Figure 6 . The blue arrow toward the left side indicates that synergism capability is more important than integration capability, and the red arrow toward the top side indicates that configuration capability is more important than integration capability.

The weighted super matrix is calculated by multiplying each element of the local priority rank vector of the unweighted super matrix by the corresponding weight of the group. If there is no comparison between two groups, the default comparison value (weights of that group) is equally important, and each column in the whole is normalized (summed to 1) after the computational processing. Finally, the weighting matrix is iterated several times, i.e., the weighting matrix is repeatedly self-multiplied until it becomes stable and then normalized. The limit super matrix is obtained from the limit matrix in the computations module of the software. The values in the limit matrix represent the global weights of the indicators in the row. Each row in the limit matrix has equal values, and the data on the column is the priority rank of the factor (node) on the left.

The algorithm is simulated using MATLAB simulation software. The number of tags is $0 \sim 1000$ and the tag sequence numbers are generated by a random sequence with a length of 96 bit. In the stage of determining the tag query prefix, a function is used to set the binary sequence to convert the decimal number, and a function is used to set the decimal number to convert the binary sequence. Since the value of the number of bits $m$ of the query prefix is related to the number of tags, $m$ is dynamically adjusted. If $m$ is too large, it will affect the energy consumption of the system and increase the burden on the system. Therefore, the optimal $\mathrm{m}$ is selected under the conditions of $1<2 \mathrm{~m}<\mathrm{L}$ and $\mathrm{n}>>2 \mathrm{~m}$ to obtain the best collision avoidance performance. Without significantly increasing the energy consumption of the system, the performance and efficiency of label recognition can be improved. The parameters are slightly adjusted in different experiments, and the average value of 100 experiments under the same conditions is chosen as the final simulation result, as shown in Figure 7.

The throughput rate of the algorithm represents the average rate at which a tag successfully passes through a channel or a node per unit time and its data information is successfully received, i.e., the product of the probability that a tag's data information is successfully read within a certain period and its input load. The throughput rate of the algorithm in this paper remains at $95 \%$ even when the number of tags is 1000 , which is a very stable trend. Although the throughput rates of the other two algorithms are also stable, the throughput rates of the DPPS algorithm are in the range of $80 \%$ to $90 \%$, and the LDPPS algorithm is stable at $90 \%$. The throughput rate of the algorithm in this paper is 5\%$15 \%$ higher than that of the DPPS algorithm and 5\% higher than that of the LDPPS algorithm.

\section{Analysis of Experimental Results}

In the simulation experiment, given the location information of 14 customer points, the crossover probability of pc0 takes the value of 0.72 , and the initial variation probability of $\mathrm{pm}$ takes the value of 0.25 , and different experimental results are obtained under different iterations, as shown in Figure 8, where the horizontal and vertical coordinates indicate the distance of relative paths, and each small cell is measured in kilometres, where the solid red dots indicate the selected customer points. The red connecting lines represent the sequence of the route through each customer point. In Figure 8 , the number of iterations is 100 , and the sum of 


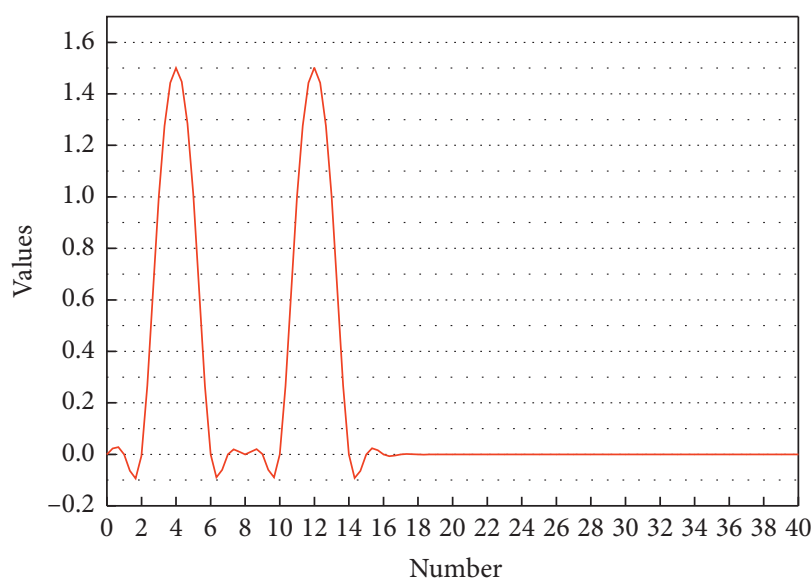

(a)

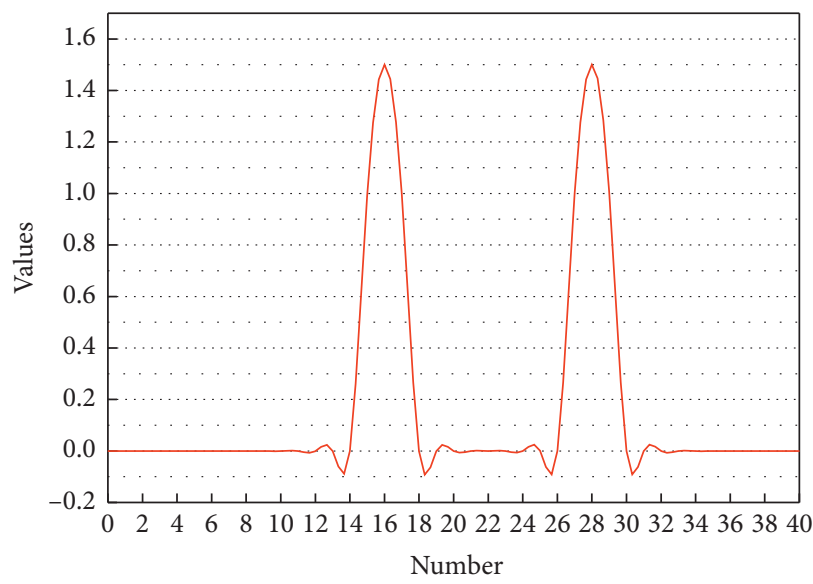

(c)

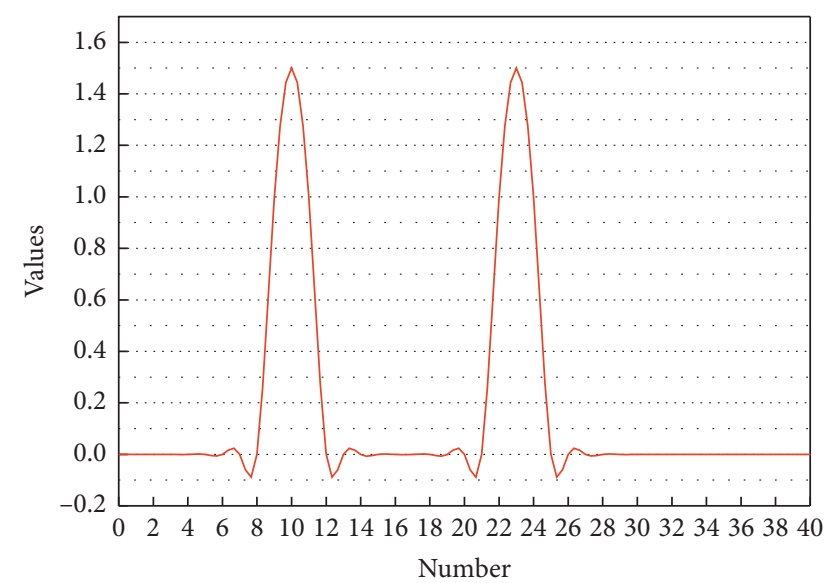

(b)

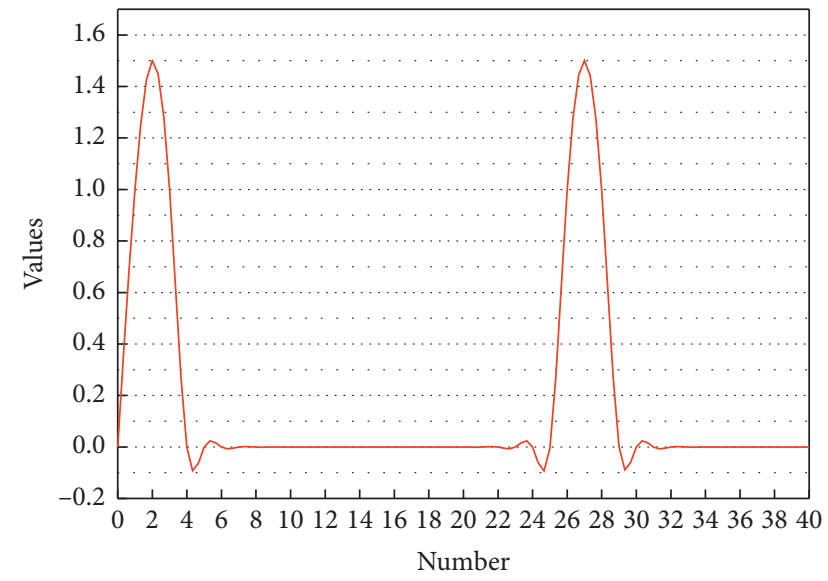

(d)

FIGURE 5: Simulation of each round refresh time of software platform transportation order library information.

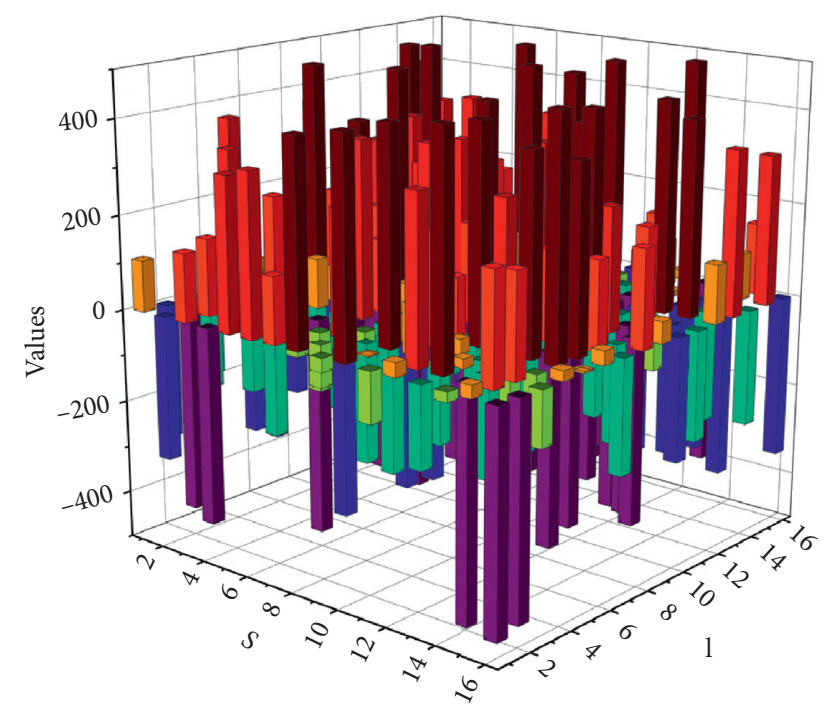

FIGURE 6: S/I/C normalized weights under A criterion.

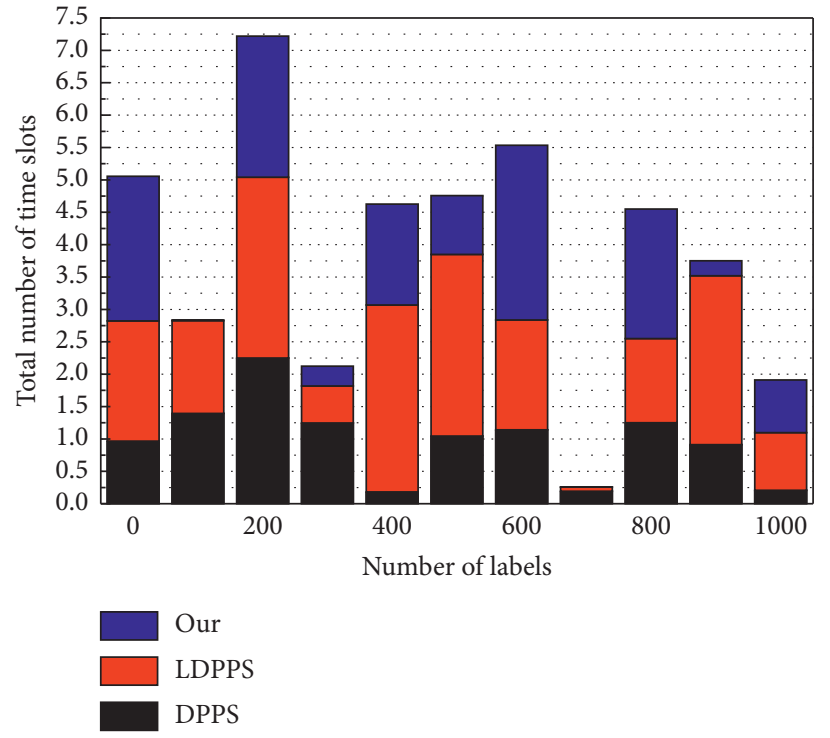

Figure 7: Throughput rate comparison. 


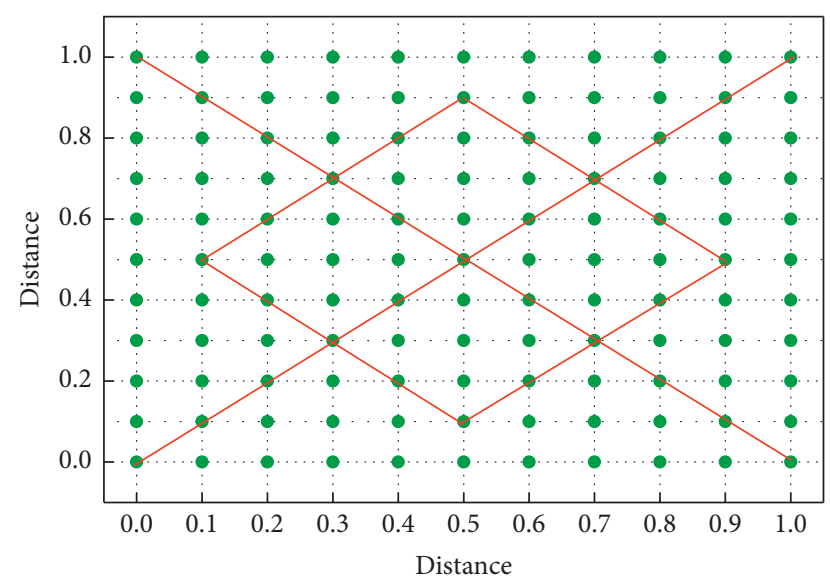

FIgURE 8: Simulation of the path optimization algorithm.

paths is 4.69. In Figure 8, the number of iterations is 500, and the sum of paths is 3.71 . From the simulation results, it can be concluded that the larger the number of iterations, the smaller the sum of paths, and the closer to the optimal path.

The data results were then compared with the results in the genetic algorithm and tested 10 times, and the results are shown in Figure 9. When the number of customer points increases, the improved genetic algorithm is slightly smaller than the genetic algorithm in terms of the average path, indicating that the improved genetic algorithm has improved performance for solving path optimization problems.

According to the specific needs of the coordination system management, the following five roles of coordination system information management are identified: Super Administrator: Manager registered user with all the rights of system operation. Order administrator: order information placement, modification, and query operations. Inventory administrator: in and out of storage operations. Distribution administrator: to not only manage the operation of the waybill but also the distribution resources and route selection operations. Order management module: by the order management personnel to modify the order information operations, such as modifying the order number, modifying the information of the recipient, and modifying the information of the sender, while confirming the status of the order. It also confirms the status of the order, such as pending, in-store, in-distribution, completed, etc. Inventory management module: the inventory manager modifies the inventory information, mainly the warehouse number, weight of goods, volume of goods, order number, in-store date, out-store date, inventory status information, etc. Distribution is a particularly important part of the coordination system, which is affected by factors such as transportation distance, tools, time, and transportation cost. The business processing part of this module mainly refers to waybill information processing and query and distribution route selection function. In the distribution route selection, the spatial information service

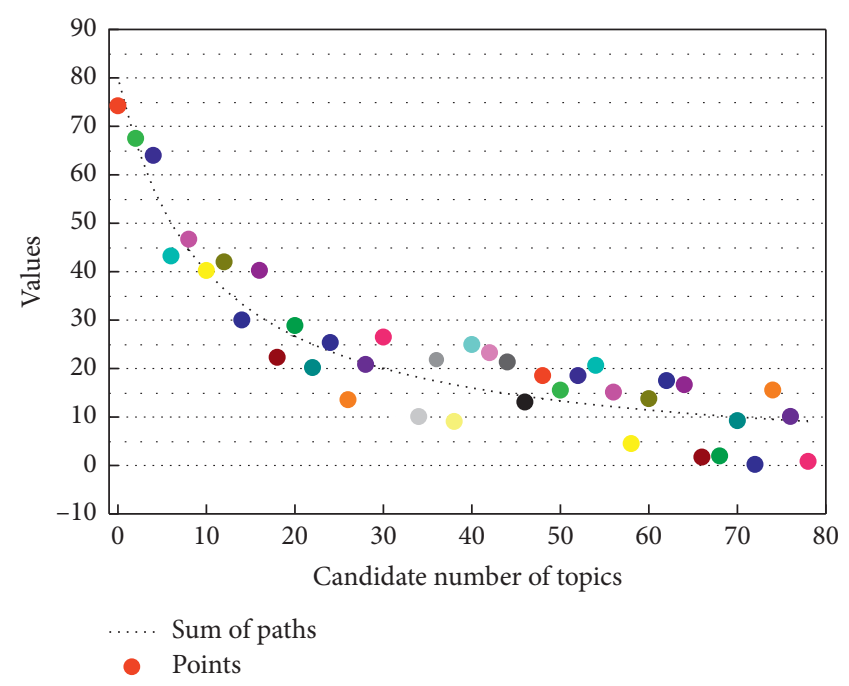

Figure 9: Algorithm comparison.

technology is mainly applied to the logistics system, its powerful spatial analysis function is used to draw the transportation path, and the logistics distribution optimization model with improved genetic algorithm provides the distribution manager with a scientific route decision basis, as shown in Figure 10.

The proposed framework of the smart supply chain is debatable. By applying emerging information technology, such as big data, Internet of Things, artificial intelligence, mobile Internet, etc., the supply chain has changed the original organization and operation of the supply chain, reconstructed new dynamic capabilities, transformed the supply chain into a smart supply chain, and improved the performance of the organization. Specifically, the emerging information technology reconstructs the dynamic collaboration capability, dynamic integration capability, and dynamic configuration capability of the supply chain. Dynamic synergy refers to the effective arrangement of tasks and resources, and the cooperation of all parties to achieve the goal, to have the ability to plan the operation of multiple resources, which is manifested as internal and external synergy, cross-professional synergy, and crossregional synergy. Dynamic configuration capability refers to the ability of business processes to adapt to changes in the supply chain environment, requiring enterprises to quickly implement the configuration of resources, such as the flexibility of personnel, equipment, processes, and resource inputs in the production process, which is manifested in the organization's flexible cooperation and agile business. Dynamic capability directly acts on the construction of an intelligent supply chain, and some scholars separate dynamic capability from enterprise organizational resources and organizational methods, and believe that dynamic capability achieves its role by changing the way of organizational operation and breaking organizational inertia. 


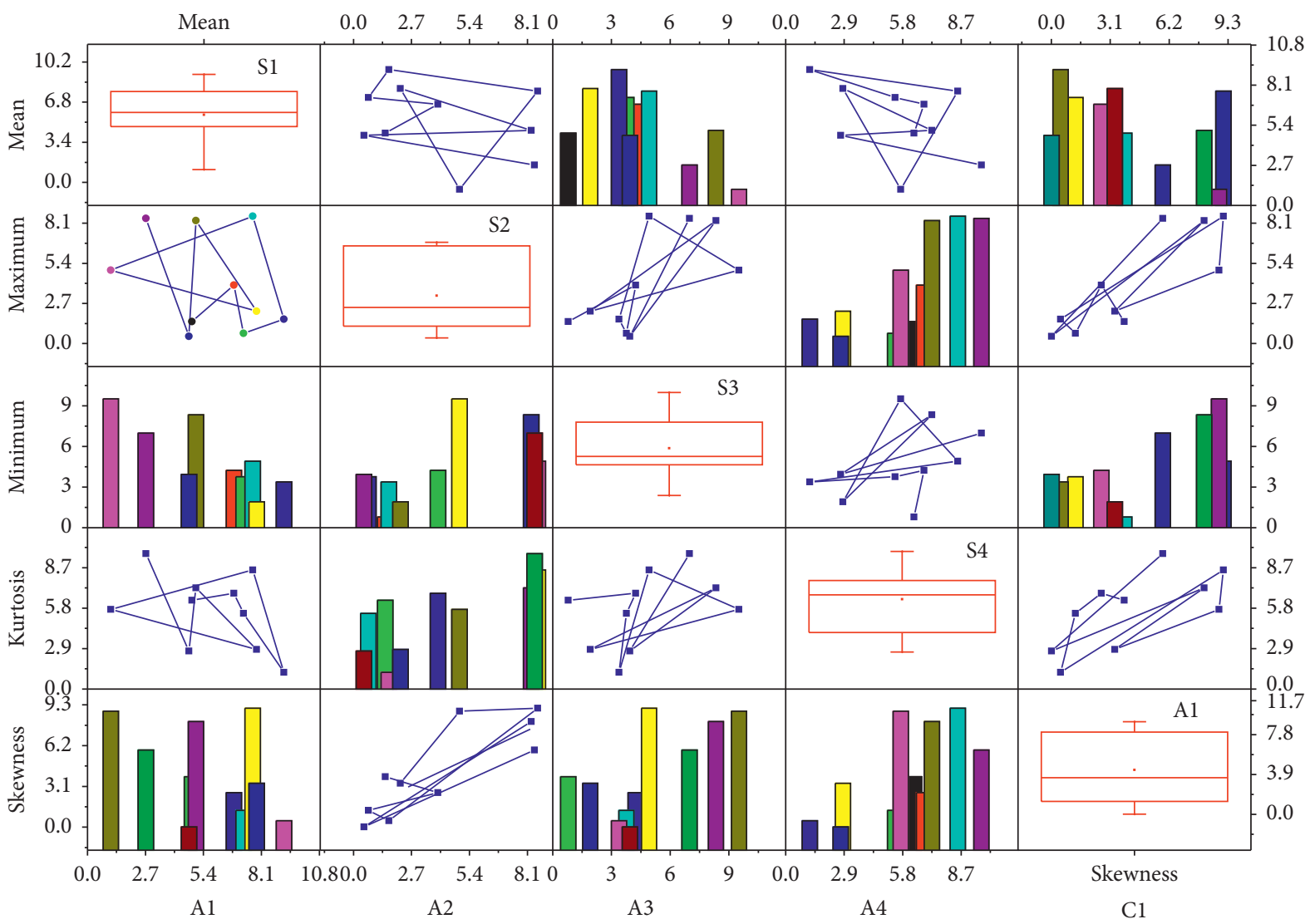

FIGURE 10: Weighted super matrix.

\section{Conclusion}

This paper introduces the overall design of the smart coordination system and the path optimization algorithm scheme. The B/S structure technology, SSM overall architecture technology, and Myasis technology used in the software implementation of the system are also introduced. A path optimization algorithm based on an improved genetic algorithm is proposed. By comparing various path optimization algorithms and investigating the path optimization problem in distribution management, it is found that the genetic algorithm has the characteristics of global convergence and high concurrency, while the Viterbi algorithm can find the optimal path by a backtracking method. A new path optimization algorithm is proposed by combining genetic algorithm and Viterbi algorithm. The design and implementation of the main functions of the intelligent logistics system are elaborated in detail; firstly, the introduction, the system business process, and the development environment are shown; this is followed by the login of this system, the interface design of the four functional modules, and the logic design of the key parts; and finally, the MySQL data operation used in this system is explained in detail. Also, the simulation results of the distribution management module reveal that the proposed path optimization algorithm has the shortest path and can effectively improve the operation efficiency of the whole coordination system. The system designed in this paper is tested for functionality and performance tests such as interface and compatibility. The test results show that the functions realized by the proposed intelligent logistics system based on the path optimization algorithm meet the basic requirements of the logistics process, and the user experience of the system interface is good, and it can run stably on the existing mainstream browsers, achieving the expected goal.

\section{Data Availability}

No datasets were generated or analyzed during the current study.

\section{Conflicts of Interest}

The author declares that there are no conflicts of interest.

\section{Acknowledgments}

The study was supported by Social Science Project of the 13th Five-Year Plan of Jilin Provincial Education Department, Research on Rural Entrepreneurship and Its Driving Factors in Changchun Economic Circle under the background of "one main and six pairs" (Grant no. JJKH20201295JY). 


\section{References}

[1] M. Mayer and A. J. Baeumner, "A megatrend challenging analytical chemistry: biosensor and chemosensor concepts ready for the internet of things," Chemical Reviews, vol. 119, no. 13, pp. 7996-8027, 2019.

[2] W. C.-C. Chu, C. Shih, W.-Y. Chou, S. I. Ahamed, and P.-A. Hsiung, "Artificial intelligence of things in sports science: weight training as an example," Computer, vol. 52, no. 11, pp. 52-61, 2019.

[3] M. Stolpe, "The internet of things," ACM SIGKDD Explorations Newsletter, vol. 18, no. 1, pp. 15-34, 2016.

[4] P. Yang, D. Stankevicius, V. Marozas et al., "Lifelogging data validation model for internet of things enabled personalized healthcare," IEEE Transactions on Systems, Man, and Cybernetics: Systems, vol. 48, no. 1, pp. 50-64, 2016.

[5] A. O. Akmandor, H. Yin, and N. K. Jha, "Smart, secure, yet energy-efficient, internet-of-things sensors," IEEE Transactions on Multi-Scale Computing Systems, vol. 4, no. 4, pp. 914-930, 2018.

[6] M. Devarajan, V. Subramaniyaswamy, V. Vijayakumar, and L. Ravi, "Fog-assisted personalized healthcare-support system for remote patients with diabetes," Journal of Ambient Intelligence and Humanized Computing, vol. 10, no. 10, pp. 3747-3760, 2019.

[7] M. Chen, Y. Zhang, M. Qiu, N. Guizani, and Y. Hao, "SPHA: smart personal health advisor based on deep analytics," IEEE Communications Magazine, vol. 56, no. 3, pp. 164-169, 2018.

[8] M. O. Al Kalaa and H. H. Refai, "Monitoring radiated coexistence testing using GMM-based classifier," IEEE Transactions on Vehicular Technology, vol. 66, no. 11, pp. 10336-10345, 2017.

[9] R. Punj and R. Kumar, "Technological aspects of WBANs for health monitoring: a comprehensive review," Wireless Networks, vol. 25, no. 3, pp. 1125-1157, 2019.

[10] M. S. Mahmud, H. Fang, and H. Wang, "An integrated wearable sensor for unobtrusive continuous measurement of autonomic nervous system," IEEE Internet of Things Journal, vol. 6, no. 1, pp. 1104-1113, 2018.

[11] J. Pagan, R. Fallahzadeh, M. Pedram et al., "Toward ultra-lowpower remote health monitoring: an optimal and adaptive compressed sensing framework for activity recognition," IEEE Transactions on Mobile Computing, vol. 18, no. 3, pp. 658-673, 2018.

[12] E. Z. Opiyo, "A feature-based approach to conceptualisation, upfront modelling, and planning for the future of complex systems," International Journal of Information Technology and Management, vol. 16, no. 1, pp. 91-112, 2017.

[13] A. A. Ugla, O. Yilmaz, and A. R. Almusawi, "Development and control of shaped metal deposition process using tungsten inert gas arc heat source in additive layered manufacturing," Proceedings of the Institution of Mechanical Engineers, Part B: Journal of Engineering Manufacture, vol. 232, no. 9, pp. 1628-1641, 2018.

[14] J. Luo and K. L. Wood, "The growing complexity in invention process," Research in Engineering Design, vol. 28, no. 4, pp. 421-435, 2017.

[15] F. Firouzi, B. Farahani, M. Ibrahim, and K. Chakrabarty, "Keynote paper: from EDA to IoT eHealth: promises, challenges, and solutions," IEEE Transactions on Computer-Aided Design of Integrated Circuits and Systems, vol. 37, no. 12, pp. 2965-2978, 2018.

[16] M. Chen, Y. Ma, J. Song, C.-F. Lai, and B. Hu, "Smart clothing: connecting human with clouds and big data for sustainable health monitoring," Mobile Networks and Applications, vol. 21, no. 5, pp. 825-845, 2016.

[17] S. Park and S. Jayaraman, "The wearables revolution and Big Data: the textile lineage," The Journal of The Textile Institute, vol. 108, no. 4, pp. 605-614, 2017.

[18] M. Goharzay, A. Noorzad, A. M. Ardakani, and M. Jalal, "Computer-aided SPT-based reliability model for probability of liquefaction using hybrid PSO and GA," Journal of Computational Design and Engineering, vol. 7, no. 1, pp. 107-127, 2020.

[19] G. Elumalai and R. Ramakrishnan, "A novel approach to monitor and maintain database about physiological parameters of (javelin) athletes using internet of things (IoT)," Wireless Personal Communications, vol. 111, no. 1, pp. 343355, 2020.

[20] U. Satija, B. Ramkumar, and M. Sabarimalai Manikandan, "Real-time signal quality-aware ECG telemetry system for IoT-based health care monitoring," IEEE Internet of Things Journal, vol. 4, no. 3, pp. 815-823, 2017.

[21] T. Saheb, "Big data analytics in the context of internet of things and the emergence of real-time systems: a systematic literature review," International Journal of High Performance Systems Architecture, vol. 8, no. 1-2, pp. 34-50, 2018.

[22] V. Miori, D. Russo, and L. Ferrucci, "Supporting active aging through A home automation infrastructure for social internet of things," Advances in Science, Technology and Engineering Systems Journal, vol. 3, no. 4, pp. 173-186, 2018.

[23] A. Aliverti, "Wearable technology: role in respiratory health and disease," Breathe, vol. 13, no. 2, pp. e27-e36, 2017.

[24] A. Manocha, R. Singh, and P. Verma, "An internet of things fog-assisted sleep-deprivation prediction framework for spinal cord injury patients," Computer, vol. 53, no. 2, pp. 46-56, 2020.

[25] D. Misic, M. Zdravkovic, M. Mitkovic, N. Vitkovic, and M. Mitkovic, "Real-time monitoring of bone fracture recovery by using aware, sensing, smart, and active orthopedic devices," IEEE Internet of Things Journal, vol. 5, no. 6, pp. 4466-4473, 2018.

[26] A. Page, S. Hijazi, S. Hijazi, D. Askan, B. Kantarci, and T. Soyata, "Support systems for health monitoring using internet-of-things driven data acquisition," Services Transactions on Services Computing, vol. 4, no. 4, pp. 18-34, 2016.

[27] A. Galli, G. Frigo, D. Chindamo, A. Depari, M. Gadola, and G. Giorgi, "Denoising ECG signal by CSTFM algorithm: monitoring during motorbike and car races," IEEE Transactions on Instrumentation and Measurement, vol. 68, no. 7, pp. 2433-2441, 2019. 